\title{
Spatial accuracy and programming of movement velocity
}

\author{
WILL A. C. SPIJKERS and ANDRIES F. SANDERS \\ Tilburg University, Tilburg, The Netherlands
}

\begin{abstract}
Two experiments in which the effect of average movement velocity on reaction time is examined in relation to spatial accuracy are reported. Experiment 1 tested the hypothesis that fast inaccurate movements are more easily accessed during response selection by varying the compatibility of the stimulus-response relation. The second experiment employed spatially accurate movements in combination with the task variables S-R compatibility and foreperiod duration. The results are consistent with a two-stage motor-preparation notion consisting of a motorprogramming stage and a program-loading stage.
\end{abstract}

Average velocity (AV) of discrete aiming movements in timing tasks appears to consistently affect reaction time (RT)-that is, the time between the onset of an imperative signal and the initiation of a movement-in that RT decreases as AV increases (Falkenberg \& Newell, 1980; Klapp \& Erwin, 1976; Spijkers \& Walter, in press). At the same time, a higher AV is accompanied by a smaller timing error (Newell, Carlton, \& Halbert, 1980). Together, these suggest that slow movements are less easily programmed (e.g., Keele, 1981). Timing of lowvelocity movements might require a more complex organization and, hence, more central processing time in advance of movement initiation.

In the above-mentioned studies, the required velocity of a particular trial was indicated by the imperative signal in order to ensure that programming of velocity occurred during $\mathrm{RT}$ rather than by preprogramming in advance of the arrival of the imperative signal (e.g., Klapp, 1977).

Evidence for preprogramming AV was indeed obtained in the study of Spijkers and Steyvers (1984, Experiment 2) and in the control task of Spijkers and Walter (in press). In the former study, the required AV was precued in advance of the imperative signal, whereas, in the latter study, AV was constant across a block of trials. In both studies, no difference was found between RT of slow and fast movements. Yet the results of the timing task of Falkenberg and Newell (1980, Experiment 2) and of Spijkers and Steyvers (1984, Experiment 1) did not support the preprogramming notion. Despite ample opportunity for preprogramming, AV had a considerable effect on RT. A major difference between the various experiments concerned the fact that, in contrast to the previously mentioned studies, these last studies required no accuracy with respect to the endpoint of the movement.

Requests for reprints should be sent to Will Spijkers, Psychology Department, Tilburg University, P.O. Box 90153, 5000 LE, Tilburg, The Netherlands.
Several studies have shown that very low spatialprecision demands reduce processing time for movements even when procedures that permit complete preprogramming of the movement are used. Thus, the no-accuracy demanding finger-lifting response in the work of Glencross (1972, Experiment 2) and of Henry and Rogers (1960) resulted in consistently shorter RTs than conditions that required more precision. Similar effects were obtained in the line-drawing task of Laszlo and Livesey (1977) and in the elbow-extension movement task of Glencross (1972, Experiment 1). Thus, the inconsistency in the findings concerning preprogramming of fast and slow movements could be due to confounding with precision requirements. Fast movements are usually less spatially precise than slow movements, unless well-controlled.

The present study explores two hypotheses that could explain a reduced RT in case of fast inaccurate movements. The first is in terms of response selection and states that abstract response codes are more easily accessed when no accuracy is demanded. The second hypothesis is based upon a two-stage response-preparation notion in which it is assumed that a motor-programming stage is followed by a program-loading stage. This last stage would translate the programmed specifications into a format suitable to the muscular system. Such a model has been proposed recently by various researchers in the area of motor control (e.g., Meyer, Yantis, Osman, \& Smith, 1984; Sternberg, Monsell, Knoll, \& Wright, 1978). From a two-stage response-preparation model, it may be inferred that a preselected program with few spatial-accuracy demands is more easily implemented into the muscular system than is a program with high spatial demands.

Selection of the appropriate response to a stimulus is usually related to S-R compatibility (e.g., Broadbent, 1971). In order to investigate the response-selection hypothesis, Experiment 1 here manipulated spatial S-R compatibility, which refers to the degree of natural association or compatibility of the spatial arrangement of the stimulus and response. As in Spijkers and Steyvers's 
(1984) Experiment 1, a 140- and a $17.5-\mathrm{cm} / \mathrm{sec}$ movement velocity were used, and were varied between blocks of trials in order to permit preprogramming. Emphasis was laid on timing accuracy, and passing the target edge was the only requirement with respect to termination. If the short RT observed in the no-accuracy conditions of previous experiments was caused by a more rapid selection of the spatial movement characteristics, then a less directly available access to the response, as in the incompatible condition, should result in a smaller velocity effect.

In Experiment 2 here, movements had equal spatialaccuracy demands. This implied only an additional requirement to the fast movement, because it was observed that the slow movement was always accurately terminated. As in Experiment 1, AV was fixed during a block of trials, so that timing as well as accuracy could be preprogrammed. It was hypothesized that if identical spatial constraints were imposed, the program-loading times would render more similar RTs for fast and slow movements. Spatial S-R compatibility was varied with the same objective in mind as in Experiment 1. Furthermore, foreperiod duration (FPD) was manipulated to vary the readiness of the motor system for adopting the preselected program (e.g., Sanders, 1980; Spijkers \& Walter, in press.)

\section{METHOD}

\section{Experiment 1}

Task and Apparatus. A visual two-choice RT task in which movement direction was the choice alternative was employed. The subjects made sliding movements across the surface of a sloping desk $\left(11^{\circ}\right)$, with an AV of either 140 or $17.5 \mathrm{~cm} / \mathrm{sec}$. A $30 \%$ deviation from these target AVs was tolerated. The sliding movement was made by the right hand through moving a stylus $(7.5 \mathrm{~g})$ from a concave circular departure point $(0.6 \mathrm{~cm}$ in diameter $)$ to a rectangular target plate $(2.4 \times$ $8.3 \mathrm{~cm})$. The apparatus was basically similar to that used by Fitts and Peterson (1964). The departure point was positioned $8.5 \mathrm{~cm}$ beneath a red warning signal located at the top of the desk. Two white lights, one on each side of the warning signal, served as imperative signals. Both to the right and to the left of the departure point was situated a metal target plate $(2.4 \times 8.3 \mathrm{~cm})$. The distance between the center of the departure point and the edge of a target plate was $7 \mathrm{~cm}$. The subjects were seated in a comfortable chair, and their right shoulders were aligned with the departure point.

A random order of signal presentation was preprogrammed on cards and read by a Graphicard reader (JNSA pattern generator PG 8). Left and right signals were equiprobable. The durations of both the warning and the imperative signals were $500 \mathrm{msec}$. A constant FPD of $2 \mathrm{sec}$ and an intertrial interval of $7 \mathrm{sec}$ were used. A reaction timer was started at the onset of the imperative signal and was stopped when the stylus left the departure point. Leaving the departure point activated a movement timer that stopped when the stylus contacted the target.

Design and Procedure. AV and S-R compatibility were varied on two levels each in a within-subjects design. In the compatible condition, the subjects moved the stylus to the target ipsilateral to the position of the imperative signal. This S-R relation was reversed in the incompatible condition. The four conditions were assigned to the subjects according to a Latin-square design. The subjects were tested on 4 consecutive days; one condition was implemented on each day. Each condition started with 120 training trials followed by two series of 90 experimental trials. There was a 10 -min rest period after the training session and between the two series.

Subjects. Four right-handed subjects, three males and one female, participated. Their ages ranged from 21 to 24 years (mean $=22.5$ years). They received Df1 7.50 per hour for their cooperation.

\section{Experiment 2}

Task and Apparatus. Compared with Experiment 1, there were some minor improvements. The departure point was reduced to $2 \mathrm{~mm}$ in order to get a more precise RT measure, and the experiment was controlled by computer (LSI 11/2). Furthermore, the width of the target was increased to $3.3 \mathrm{~cm}$ in order to facilitate accurate ending at the target in the fast movement condition.

Design and Procedure. The independent variables AV and S-R compatibility were identical to those in Experiment 1 . In addition, there were two FPD durations, that is, a 2- or a 7-sec interval between the onset of the warning and of the imperative signal.

AV, S-R compatibility, and FPD were varied across blocks, whereas movement direction was determined randomly at each trial. Each subject completed two sessions. The sessions, run on different days, both consisted of four series of 50 trials. Between series, there was a short break of $5 \mathrm{~min}$. During a session, AV was fixed. For half the subjects, $A V$ in the first session was either fast or slow. FPD was counterbalanced over the four series of each session according to a BAAB or ABBA sequence. Spatial S-R compatibility was kept constant during two consecutive series. Due to an assignment error, four of the six subjects received the compatibility-incompatibility sequence, and only two subjects received the incompatibility-compatibility sequence. In the second session, the order of conditions was similar to that in the first session.

Subjects. Six subjects, ranging in age from 21 to 25 years (mean = 22.9 years), participated. They were right-handed and received Df1 7.50 per hour for their participation.

It had become evident in Experiment 1 that it was not easy to accomplish the fast movement without overshooting the target. It was decided, therefore, to select subjects on the basis of performance in this specific condition in order to avoid lengthy and frustrating training sessions. The criterion for participation was a score of 9 of 10 correct trials within a training phase of 100 trials. A correct trial was defined as both fast and accurate. Of the eight subjects tested, two could not fulfill this requirement.

\section{RESULTS}

\section{Experiment 1}

Errors. Velocity errors-that is, movements outside the speed tolerance limits-were more frequent in the high$(8.8 \%)$ than in the low- $(<1 \%)$ velocity condition. Accuracy of timing was less for the fast movements, since the variable error expressed as a percentage of the velocity of the correct movements was higher in the high$(22.7 \%)$ than in the low- $(11.8 \%)$ velocity condition. The percentage of incorrect reactions, that is, wrong-direction and extremely fast $(<100 \mathrm{msec})$ or slow $(>800 \mathrm{msec})$ reactions, was slightly higher in the incompatible condition (fast: $3.5 \%$; slow: $2.0 \%$ ) than in the compatible condition (fast: $2.0 \%$; slow: $1.3 \%$ ).

Reaction time. RTs for incorrect reactions and velocity errors were discarded from the analysis. A pooled 2 $\times 2 \times 2(\mathrm{AV} \times \mathrm{S}-\mathrm{R}$ compatibility $\times$ series $)$ ANOVA was carried out. The factor series was not significant $[F(1,21)=0.14]$. Averaged over series, mean RTs for high and low movement velocity were, respectively, 287 and $346 \mathrm{msec}$ in the compatible condition and 325 and $381 \mathrm{msec}$ in the incompatible condition. Low movement velocity increased RT considerably $-57 \mathrm{msec}[\mathrm{F}(1,21)=$ 73.8, $\mathrm{p}<.001$ ], which replicates Spijkers and Steyvers's (1984) Experiment 1. Incompatibility of the relation between the signal and direction to move prolonged the RT by $36 \mathrm{msec}$ on the average $[\mathrm{F}(1,21)=27.4, \mathrm{p}<.001]$. There was no interaction between the effects of $\mathrm{AV}$ and S-R compatibility $[\mathrm{F}(1,21)=.05]$. 
Table 1

Mean Reaction Times (in Milliseconds) as a Function of Average Velocity, Foreperiod (FPD; in Seconds), and S-R Compatibility

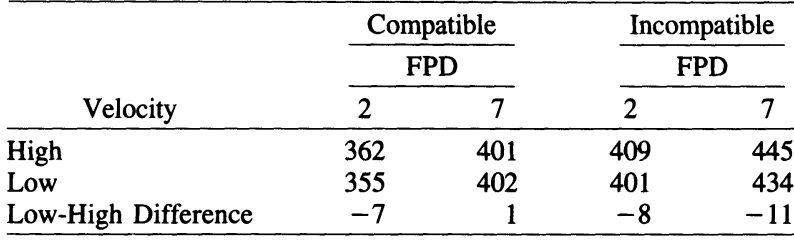

In the high-velocity condition, actual AVs were slightly faster than had been instructed -145.8 and $166.6 \mathrm{~cm} / \mathrm{sec}$ for the compatible and incompatible movements, respectively. Low velocity was attained quite well $-17.7 \mathrm{~cm} / \mathrm{sec}$ in both compatibility conditions.

\section{Experiment 2}

Reaction times. An ANOVA was carried out with subjects, S-R compatibility, FPD, AV, movement direction, and series as factors. Only S-R compatibility $[\mathrm{F}(1,5)=$ 17.6, $\mathrm{p}<.01]$ and FPD $[\mathrm{F}(1,5)=71.3, \mathrm{p}<.001] \mathrm{had}$ significant main effects. Table 1 shows the mean correct RTs averaged over subjects, series, and direction.

An interaction of S-R compatibility, FPD, and series was found $[F(1,5)=15.4, p<.05]$. Inspection of the data showed that in the first series the RT in the incompatible condition was longer at the short than at the long FPD (55 vs. $33 \mathrm{msec}$ ), whereas this differential effect was almost absent in the second series (41 vs. $33 \mathrm{msec}$ ).

Movement times. Apart from AV $[\mathrm{F}(1,5)=1806.3$, $\mathrm{p}<.001$ ], no independent variable affected movement duration. Across conditions, actual AV varied between 129.6 and $134.6 \mathrm{~cm} / \mathrm{sec}$ for the fast movements and between 17.8 and $18.4 \mathrm{~cm} / \mathrm{sec}$ for the slow movements.

Reaction errors. Reaction errors were equally distributed over fast $(6.9 \%)$ and slow $(7.2 \%)$ movements. Average error percentages were $4.8 \%$ in the compatible condition and $8.9 \%$ in the incompatible condition.

Velocity errors. More velocity errors were made in the fast- $(16.1 \%)$ than in the slow- $(8.1 \%)$ movement condition, but this difference was only marginally significant $[F(1,5)=4.7, p<.1]$. Accuracy of timing was better for the fast movements, since variable error was slightly lower in the fast $(9.3 \%)$ than in the slow $(11.5 \%)$ condition. Incorrect RTs and RTs followed by a velocity error were discarded from analysis.

Overshoots. Overshoots occurred only in the highvelocity condition and were equally distributed over the conditions. This means that accuracy was not differentially traded for speed among the experimental conditions. Compared with the pretest, the error score increased from $10 \%$ to $24.5 \%$ in the experimental sessions.

\section{DISCUSSION}

With respect to spatial S-R compatibility, the results of both experiments are similar in that $S-R$ compatibility and AV had independent effects on RT. Both findings argue against the hypothesis that easy ac- cess of the response is the source of the rapid initiation of fast inaccurate movements. In addition, Spijkers and Walter (in press) have also found additive effects of semantic S-R compatibility and AV. Thus, there is convergent evidence in favor of the notion that response selection and motor programming constitute separate stages in the reaction process.

Furthermore, programming of velocity is not influenced by changes in motor-response readiness, as FPD is assumed to bring about (Sanders, 1980). Additivity of the effects of AV and FPD has now been observed for inaccurate fast movements (Experiment 1 of Spijkers \& Steyvers, 1984), for accurate fast movements the velocity of which can be preprogrammed (this study), and for accurate fast movements the velocity of which must be programmed during the RT (Spijkers \& Walter, in press). Thus, the conclusion appears justified that implementation of a (pre-)constructed motor program does not depend on the state of motor readiness.

Comparison of RT to fast movements between Experiments 1 and 2 shows that, when positional accuracy is demanded, RT for fast movements increases. This indicates that additional processing is required when initiating a fast, precisely ending movement. This is consistent with a two-stage model of response preparation in which a programloading stage follows a motor-programming stage. In this model, motor programming is assumed to be concerned with the specification of the motor-control parameters such as velocity and direction, which are open to preprogramming. The program-loading stage translates the specifications into a format appropriate to the muscular system. Loading time depends on the accuracy demands of the ensuing movement. It cannot be preprogrammed, but is always performed during the RT. The inability to preprogram may seem inefficient, but may provide greater versatility, since the same program can be adopted when loading different muscular systems.

The finding that the large timing error observed for the fast inaccurate movements in Experiment 1 was strongly reduced when the movements were more accurately performed (Experiment 2 ) is not inconsistent with the proposed model. A longer program-loading time might also have beneficial effects on timing accuracy.

Posthoc, the two-stage model can account for those studies in which inaccurate movements are initiated faster than accurate movements (e.g., Falkenberg \& Newell, 1980), even when movement timing can be preprogrammed (e.g., Experiment 1 of this study). Moreover, it can accommodate the effects of AV of accurate movements on RT irrespective of whether (1) the velocity is indicated by the imperative signal (Spijkers \& Steyvers, 1984; Spijkers \& Walter, in press) or (2) velocity can be preprogrammed (Experiment 2 of this study; Spijkers \& Steyvers, 1984; Spijkers \& Walter, in press). One further test of the model would be a comparison of RT for inaccurate movements the timing of which either can or cannot be preprogrammed. In this case, loading times are similar for both conditions, so that RT should be longer when preprogramming is impossible.

The two-stage model should be regarded as particularly pertinent to movements that are subject to open loop control. If accuracy can be adjusted during the movement, the program-loading stage is supposed to be only minimally involved in shaping the movement during RT. This means that a small effect of accuracy on RT is expected in more closedloop controlled movements, which actually was the case in the work of Fitts (e.g, Fitts \& Peterson, 1964).

\section{REFERENCES}

Broadbent, D. E. (1971). Decision and stress. New York: Academic Press.

FALKENBERG, L. E., \& Newell, K. M. (1980). Relative contribution of movement time, amplitude, and velocity to response initiation. Journal of Experimental Psychology: Human Perception and Performance, 6, 760-768.

FitTs, P. M., \& Peterson, J. R. (1964). Information capacity of discrete motor responses. Journal of Experimental Psychology, 67, 103-112.

GLeNCross, D. J. (1972). Latency and response complexity. Journal of Motor Behavior, 4, 251-256.

HENRY, F. M., \& RoGERS, D. E. (1960). Increased response latency 
for complicated movements and a "memory drum" theory of neuromotor reaction. Research Quarterly, 31, 448-458.

KeELE, S. W. (1981). Behavioral analysis of motor control. In V. Brooks (Ed.), Motor control: Handbook of physiology (Vol. 2, pp. 13911414). Baltimore, MD: Williams and Wilkins.

KLAPP, S. T. (1977). Reaction time analysis of programmed control. Exercise and sport sciences reviews, pp. 231-253.

KLAPP, S. T., \& ERWIN, C. I. (1976). Relation between programming time and duration of the response being programmed. Journal of Experimental Psychology: Human Perception and Performance, 2, 591-598.

LASZlo, J. I., \& LiveSEY, J. P. (1977). Task complexity, accuracy, and reaction time. Journal of Motor Behavior, 9, 171-177.

Meyer, D. E., Yantis, S., Osman, A., \& Smith, K. J. E. (1984). Discrete versus continuous models of response preparation: A reaction time analysis. In S. Kornblum \& J. Requin (Eds.), Preparatory states and processes (pp. 69-94). Hillsdale, NJ: Erlbaum.

Newell, K. M., Carlton, M.J., \& Halbert J. A. (1980). Movement velocity as a factor in movement timing accuracy. Journal of Motor Behavior, 12, 47-56.

SANDERS, A. F. (1980). Stage analysis of reaction process. In G. E. Stelmach \& J. Requin (Eds.), Tutorials in motor behavior (pp. 331354). New York: North-Holland.

SpiJkers, W. A. C., \& STeyvers, F. P. J. M. (1984). Specification of direction and duration during programming of discrete sliding movements. Psychological Research, 46, 59-71.

SpiJKers, W. A. C., \& Walter, A. (in press). Response processing stages in choice reactions. Acta Psychologica.

Sternberg, S., Monsell, S., Knoll, R., \& Wright, C. E. (1978). The latency and duration of rapid movement sequences: Comparisons of speech and typewriting. In G. E. Stelmach (Ed.), Information processing in motor control and learning (pp. 117-152). New York: Academic Press.

(Revision received for publication October 19, 1984.) 